\title{
An LCMS-based untargeted metabolomics protocol for cochlear perilymph: highlighting metabolic effects of hydrogen gas on the inner ear of noise exposed Guinea pigs
}

\author{
Kristian Pirttilä ${ }^{1}$. Pernilla Videhult Pierre ${ }^{2}$. Jakob Haglöf ${ }^{1}$. Mikael Engskog ${ }^{1}$ - Mikael Hedeland ${ }^{1}$. Göran Laurell ${ }^{3}$. \\ Torbjörn Arvidsson ${ }^{1} \cdot$ Curt Pettersson ${ }^{1}$
}

Received: 26 April 2019 / Accepted: 25 September 2019 / Published online: 5 October 2019

(c) The Author(s) 2019

\begin{abstract}
Introduction Noise-induced hearing loss (NIHL) is an increasing problem in society and accounts for a third of all cases of acquired hearing loss. NIHL is caused by formation of reactive oxygen species (ROS) in the cochlea causing oxidative stress. Hydrogen gas $\left(\mathrm{H}_{2}\right)$ can alleviate the damage caused by oxidative stress and can be easily administered through inhalation.

Objectives To present a protocol for untargeted metabolomics of guinea pig perilymph and investigate the effect of $\mathrm{H}_{2}$ administration on the perilymph metabolome of noise exposed guinea pigs.

Methods The left ear of guinea pigs were exposed to hazardous impulse noise only (Noise, $\mathrm{n}=10$ ), noise and $\mathrm{H}_{2}$ (Noise $+\mathrm{H}$, $\mathrm{n}=10$ ), only $\mathrm{H}_{2}(H 2, \mathrm{n}=4)$, or untreated (Control, $\mathrm{n}=2$ ). Scala tympani perilymph was sampled from the cochlea of both ears. The polar component of the perilymph metabolome was analyzed using a HILIC-UHPLC-Q-TOF-MS-based untargeted metabolomics protocol. Multivariate data analysis (MVDA) was performed separately for the exposed- and unexposed ear. Results MVDA allowed separation of groups Noise and Noise $+\mathrm{H} 2$ in both the exposed and unexposed ear and yielded 15 metabolites with differentiating relative abundances. Seven were found in both exposed and unexposed ear data and included two osmoprotectants. Eight metabolites were unique to the unexposed ear and included a number of short-chain acylcarnitines.

Conclusions A HILIC-UHPLC-Q-TOF-MS-based protocol for untargeted metabolomics of perilymph is presented and shown to be fit-for-purpose. We found a clear difference in the perilymph metabolome of noise exposed guinea pigs with and without $\mathrm{H}_{2}$ treatment.
\end{abstract}

Keywords Metabolomics $\cdot$ NIHL $\cdot$ In vivo $\cdot$ Noise-induced hearing loss $\cdot$ LCMS $\cdot$ Perilymph

\begin{tabular}{|c|c|c|c|}
\hline \multicolumn{2}{|c|}{ Abbreviations } & BPI & Base peak ion \\
\hline \multicolumn{2}{|r|}{ Auditory brainstem response } & CCR & Correct classification rate \\
\hline \multicolumn{2}{|r|}{ Acetonitrile } & CSF & Cerebrospinal fluid \\
\hline & & $\mathrm{H}_{2}$ & Hydrogen gas \\
\hline & & ESI & Electrospray ionization \\
\hline \multirow{3}{*}{\multicolumn{2}{|c|}{$\begin{array}{l}\text { Electronic supplementary material The online version of this } \\
\text { article (https://doi.org/10.1007/s11306-019-1595-1) contains } \\
\text { supplementary material, which is available to authorized users. }\end{array}$}} & LCMS & Liquid chromatography mass spectrometry \\
\hline & & LOOCV & Leave-one-out cross validation \\
\hline & & MVDA & Multivariate data analysis \\
\hline \multirow[t]{2}{*}{$\square$} & Kristian Pirttilä & NIHL & Noise-induced hearing loss \\
\hline & kristian.pirttila@ilk.uu.se & OPLS-DA & Orthogonal projection to latent structures \\
\hline 1 & $\begin{array}{l}\text { Department of Medicinal Chemistry, Uppsala University, } \\
\text { Uppsala, Sweden }\end{array}$ & PCA & $\begin{array}{l}\text { discriminant analysis } \\
\text { Principal component analysis }\end{array}$ \\
\hline \multirow[t]{3}{*}{2} & Division of Audiology, Department of Clinical Science, & QC & Quality control \\
\hline & Intervention and Technology, Karolinska Institutet, & QTOF & Quadrupole time of flight \\
\hline & Stockholm, Sweden & ROS & Reactive oxygen species \\
\hline 3 & Department of Surgical Science, Uppsala University, & $\mathrm{S} / \mathrm{N}$ & Signal to noise \\
\hline
\end{tabular}


SUS plot Shared and unique structures plot

UPLC Ultra performance liquid chromatography

\section{Introduction}

Noise induced hearing loss (NIHL) is a common disorder that accounts for a third of all cases of acquired hearing loss worldwide (Le et al. 2017; Sha and Schacht 2017). The cochlea is a structure of the inner ear that harbor the sensory cells of hearing and is filled with endolymph and perilymph. Noise exposure can lead to pathological changes in the cochlea causing hearing impairment. One key mechanism is the formation of reactive oxygen species (ROS) leading to oxidative stress in the inner ear (Henderson et al. 2006; Le et al. 2017). The ROS may leak into the surrounding tissue, causing widespread damage over the course of several days (Le et al. 2017). In the past, hydrogen gas $\left(\mathrm{H}_{2}\right)$ has been shown to reduce oxidative stressinduced effects in a number of organ systems (Fukuda et al. 2007; Huang et al. 2011). In addition, $\mathrm{H}_{2}$ has been shown to reduce hearing loss due to noise exposure (Lin et al. 2011; Kurioka et al. 2014) and cisplatin ototoxicity (Qu et al. 2012; Kikkawa et al. 2014; Fransson et al. 2017). However, more knowledge about the molecular mechanisms surrounding the protective effect is needed.

Blood is a common sample material in metabolomics studies owing to the relative ease of sampling. As blood permeates the entire body it comes in contact with every organ. Subsequently, the blood metabolite composition represents an approximate average of the metabolic states of the whole body (Psychogios et al. 2011). When studying inherently localized biological effects the use of localized tissue or fluids sampled in proximity to the afflicted region is preferable as these are more representative of the system being studied. In that respect, cochlear perilymph is in close contact with the sensory cells of hearing, the inner and outer hair cells, making it highly suitable for studying acquired hearing loss. However, perilymph sampling cannot be performed in humans without causing iatrogenic hearing loss. It is also challenging in experimental animals since only a very small volume can be drawn to avoid the risk of contamination with cerebrospinal fluid (CSF) (Hara et al. 1989). In this work, scala tympani perilymph from guinea pigs, a frequently used species in hearing research, was analyzed as this can be safely sampled up to $1 \mu \mathrm{L}$ using available surgical techniques. As a consequence of the difficulty in obtaining samples, perilymph is a relatively unknown sample material in terms of bioanalysis. Most of the literature on the composition of human perilymph has been focused on its proteome content and is limited to patients undergoing surgical interventions (Lysaght et al. 2011; Schmitt et al. 2017; Edvardsson Rasmussen et al. 2018; Palmer et al. 2018). Some attention has been directed at characterizing the small metabolites of perilymph using mainly HPLC with fluorescence detection (Drescher, Medina and Drescher 1981; Medina and Drescher 1981; Scheibe and Haupt 1985; Bobbin and Fallon 1992). The qualitative composition of perilymph, in terms of proteins and small metabolites, is comparable to that of both CSF and blood plasma, whereas the relative concentrations differ widely between the three fluids (Scheibe and Haupt 1985; Leary Swan et al. 2009; Fujita et al. 2015). Notably, Fujita et al. (2015) used a GCMS-based metabolomics protocol to study the effect of noise on the metabolome of the inner ear fluid of guinea pigs. They found ten metabolites that were significantly altered in concentration in inner ear fluid, whereas no altered metabolite concentrations were found in blood. Recently, Mavel et al. (2018) used LCMS to characterize the metabolites of human perilymph in patients undergoing cochlear implantation. Most likely due to the limited availability of perilymph from healthy subjects, no control samples were included in their study, and no group comparison was made. However, they were able to identify a large number of compounds from various biological pathways using their developed protocol.

LCMS, in contrast to GCMS, allows the detection of thermally labile and highly polar compounds without need of derivatisation. To the best of our knowledge, untargeted metabolomics using LCMS has not previously been applied to study hearing loss. Furthermore, the use of $\mathrm{H}_{2}$ to attenuate NIHL has not been studied at the metabolite level. To that end, we present an LCMS-based untargeted metabolomics protocol for perilymph applied to a guinea pig model of the attenuating effect of $\mathrm{H}_{2}$ on NIHL.

\section{Materials and methods}

\subsection{Chemicals}

All water was purified using a Milli- $\mathrm{Q}^{\mathrm{TM}}$ water system from MilliPore (Bedford, MA, USA). Ammonium formate (LCMS-grade) and formic acid (LCMS-grade) were obtained from Sigma-Aldrich (Steinheim, Germany). Acetonitrile (LCMS-grade) was purchased from Fisher Scientific (Zurich, Switzerland). Acetonitrile (ACN) used for protein precipitation was kept on ice throughout the sample preparation procedure. Butyryl-L-carnitine, creatine, $O$-acetylL-carnitine hydrochloride, and stachydrine-(dimethyl- ${ }^{13} \mathrm{C}_{2}$ ) monohydrate were obtained from Sigma-Aldrich. 


\subsection{Software}

LCMS equipment was controlled using MassLynx (ver. 4.1, Waters Corp., Milford, USA). Databridge (ver. 3.5, Micromass UK Ltd., Manchester, England) was used to convert .raw files to .cdf files. All data preprocessing and univariate analysis were performed in the $\mathrm{R}$ statistical language environment (ver. 3.4.2). Peak picking, feature detection, retention alignment, and peak filling were performed using the XCMS R-package (Smith et al. 2006). The IPO R package (Libiseller et al. 2015) was used to find pseudo-optimal XCMS parameters. Adduct annotation was performed using the CAMERA R-package (Kuhl et al. 2012; Kuhl et al. 2014). Multivariate data analysis (MVDA) was performed in SIMCA (ver. 14.1, Umetrics AB, Umeå, Sweden).

\subsection{In vivo procedures}

All animal procedures and results of auditory brainstem response (ABR) assessment and histology are reported in full elsewhere (Videhult Pierre et al. Hydrogen gas inhalation attenuates acute noise trauma. A preclinical in vivo study, in manuscript). In short, the left ear of guinea pigs was exposed to hazardous impulse noise $(156 \mathrm{~dB}, 400$ impulses, $\sim 4 \mathrm{~min}$ ) only (Noise; $\mathrm{n}=10$ ) immediately followed by inhalation of $\mathrm{H}_{2}$ ( $2 \%$ in air, $60 \mathrm{~min}$; Noise $+\mathrm{H}_{2}$; $\mathrm{n}=10)$. Additional animals received only $\mathrm{H}_{2}(H 2 ; \mathrm{n}=4)$ or no treatment at all (Control; $\mathrm{n}=2$ ). After ABR recordings at day $4,1 \mu \mathrm{L}$ of scala tympani perilymph was sampled using calibrated capillary tubes from the basal turn of each cochlea (Hellberg et al. 2013) added to a pre-chilled vial containing $19.0 \mu \mathrm{L} \mathrm{H}_{2} \mathrm{O}$, and stored at $-80{ }^{\circ} \mathrm{C}$ until further treatment.

\subsection{Sample preparation}

Sample preparation was performed in batches of 30 samples. Batches were randomized to achieve an even distribution of sample groups across all batches. Protein precipitation was performed on ice by addition of $20.0 \mu \mathrm{L}$ ice-cold acetonitrile $(4: 1, \mathrm{v} / \mathrm{v})$ to $5.0 \mu \mathrm{L}$ sample aliquots. A quality control (QC) sample was prepared as a pooled mixture of sample aliquots and subjected to the same protein precipitation protocol. Samples were precipitated at $8{ }^{\circ} \mathrm{C}$ for $30 \mathrm{~min}$ and centrifuged $\left(21,000 \times g, 4{ }^{\circ} \mathrm{C}, 15 \mathrm{~min}\right)$. The supernatant was kept at $-80{ }^{\circ} \mathrm{C}$ for no more than 7 days. Before analysis all samples were analyzed without further treatment. Samples were kept at $4{ }^{\circ} \mathrm{C}$ throughout the analytical run.

\subsection{LC-ESI-Q-TOF MS sample analysis}

Chromatographic separation and detection was achieved using an ACQUITY I-class UPLC system (Waters Corp.,
Milford, USA) coupled to a Synapt G2-S QTOF HRMS instrument (Waters).

\subsubsection{Chromatographic separation}

Chromatographic separation was performed in HILIC mode using a Waters BEH Amide column $(50 \times 2.1 \mathrm{~mm}$ i.d., $1.7 \mu \mathrm{m}$ particle size, $100 \AA$ pore size) fitted with a Waters VanGuard BEH Amide $(5 \times 2.1 \mathrm{~mm}$ i.d., $1.7 \mu \mathrm{m}$ particle size, $100 \AA$ pore size) pre-column. Mobile phase A was prepared by addition of $100 \mathrm{~mL}$ ammonium formate buffer (200 $\mathrm{mM}$ in water, adjusted to $\mathrm{pH} 3$ using formic acid) to $1900 \mathrm{~mL}$ ACN. Mobile phase B was prepared by addition of $100 \mathrm{~mL}$ ammonium formate buffer $(200 \mathrm{mM}$ in water, $\mathrm{pH} 3$ ) and $100 \mathrm{~mL}$ ACN to $1800 \mathrm{~mL}$ water. Strong- and weak wash solutions were 10:90 and 95:5 ACN:water (v/v), respectively. All mixtures were degassed by ultrasonication for $20 \mathrm{~min}$. Elution was performed using a gradient from 0 to $61 \% \mathrm{~B}$ over 14 min using a non-linear concave gradient (setting 8 in MassLynx, see supporting info for a definition) and kept at $61 \% \mathrm{~B}$ for 2.7 min followed by a gradient back to $0 \% \mathrm{~B}$ over $0.3 \mathrm{~min}$ and re-equilibration at $0 \% \mathrm{~B}$ for $6 \mathrm{~min}$. The column temperature was $40{ }^{\circ} \mathrm{C}$. Sample injection volume was $4 \mu \mathrm{L}$.

\subsubsection{MS detection}

Data were collected in ESI+ and ESI- mode in two different analytical runs. Mass calibration was performed according to manufacturer guidelines using sodium formate. Centroided mass data were acquired in resolution mode. For a full list of tune settings, see Supplementary Material. Internal mass calibration was achieved using a solution of leucineenkephalin $(0.2 \mathrm{nM}$ in $1: 1 \mathrm{ACN}$ :water $+0.1 \%$ formic acid $)$ infused at $10 \mu \mathrm{L} / \mathrm{min}$ and sampled every $15 \mathrm{~s}$ with a scan time of $0.3 \mathrm{~s}$. Data was acquired in $M \mathrm{~S}^{\mathrm{E}}$ mode consisting of a full scan step between $m / z 50-800$ with a 1.0 s scan time followed by a high energy scan between $m / z 50-800$ with a $1.0 \mathrm{~s}$ scan time and transfer collision energy ramp from 20 to $45 \mathrm{eV}$.

\subsection{Quality control}

\subsubsection{Pre-analytical quality control}

System suitability was assessed using an internal protocol and comparison to historic data for the instrument. In short, a solution of paracetamol, leucine-enkephaline, and meloxicam was injected and the result plotted as response intensity and mass accuracy as a function of time.

Prior to starting the analytical run the instrument was conditioned by repeated injection of the QC sample (Sangster et al. 2006; Gika et al. 2007). Stability was assessed in 
two ways: using (i) a targeted protocol of a set of known endogenous compounds detected in the QC sample, and (ii) an untargeted protocol using PCA of data generated from the conditioning injections using MarkerLynx (Waters). The drift in retention times and peak intensities were monitored until the RSD of peak intensities and retention times was $<10 \%$ and $<1 \%$, respectively across five consecutive injections. For a full list of substances used for monitoring the conditioning, see Supplementary Material. Principal component analysis (PCA) was performed successively and the scores plot monitored until convergence of the points in the first and second component.

\subsubsection{Inter-analytical quality control}

The analytical run was initiated by injecting the QC sample. The analytical run then consisted of seven sample injections followed by a QC sample injection until all samples had been analyzed. Throughout the analytical run the same set of metabolites used to monitor the conditioning step was monitored in the QC sample injections. Retention time and peak intensity were determined to assess the presence of any sudden changes or major drift in instrument performance during the analytical run.

\subsubsection{Post-analytical quality control}

To assess the residual systematic drift and instrumental noise following data pretreatment, the full dataset including the QC sample injections was analyzed using PCA. This allows the existence and relative scale of such variation to be assessed in relation to the biological variation between samples as well as the detection of potentially outlying samples or features. Systematic error in peak intensities originating from instrument performance drift was assessed from the scores plot by the relative spatial distribution of the QC sample injections in relation to the distribution of study samples. The QC sample injections should cluster tightly at the center of the study samples.

\subsection{Data preprocessing}

XCMS parameters were optimized using the IPO package and adjusted to be slightly more inclusive. CAMERA was used for adduct annotation using an extended ruleset (Stanstrup et al. 2013). See Supplementary Information for the initial parameters used with IPO, the final pseudo-optimized
XCMS parameters, and the CAMERA parameters. Peak intensity normalization was performed by calculating the average feature intensity across all QC sample injections and calculating the feature-wise fold change of every sample to this average QC sample injection. Each sample was then normalized by dividing each feature intensity with the median of all feature-wise fold changes to the average QC sample injection (Dieterle et al. 2006; Veselkov et al. 2011; Wu and $\mathrm{Li}$ 2016). Features with a retention time of $60-800 \mathrm{~s}$ and a peak area RSD $<30 \%$ in the QC sample injections were kept.

\subsection{Multivariate data analysis and feature selection}

PCA was performed on pareto-scaled data and was used to detect potential outliers, assess data quality, and visualize major structures in the data. Orthogonal projection to latent structures discriminant analysis (OPLS-DA) was used to model group membership in samples to find features important to group classification. The models used for feature selection was fitted on the full data set using 7-fold cross validation and overfit was assessed based on the PCA analysis, R2 and Q2 metrics, permutation tests $(\mathrm{n}=500)$, as well as CV-ANOVA (Eriksson, Trygg and Wold 2008) in SIMCA. The classification error was estimated by leave-oneout cross validation (LOOCV) using 7-fold cross validation to fit models to the training sets and is further described in the Supporting Information. The correct classification rate (CCR) was determined as the ratio of the correctly classified samples over the total number of samples. Feature selection and comparison of models fitted on data from the left- and right ear was performed using the S- and SUS-plot (Wiklund et al. 2008) in SIMCA. A feature was selected for further study if it had a $\mid p($ corr) $\mid>0.5$ or belonged to a cluster centered above this value. Adducts and fragments with comparable Ip(corr)l-values were also included in the selection based on adduct annotation from the CAMERA package.

\subsection{Peak verification of selected features}

Each feature selected in the MVDA step was subjected to manual inspection of the raw data in terms of peak shape and signal-to-noise $(\mathrm{S} / \mathrm{N})$ ratio. A peak was considered valid if it had a S/N over 10, an approximate Gaussian peak shape and no disturbing isobaric coelutions or irregularities in the peak that could make integration highly variable. Furthermore, adducts and fragments were manually verified by comparison of peak shape and retention times, grouping features with overlapping peaks and $\mathrm{m} / \mathrm{z}$ differences corresponding to adducts and known fragments. Each peak was also crossreferenced with the original dataset to verify that it was not 

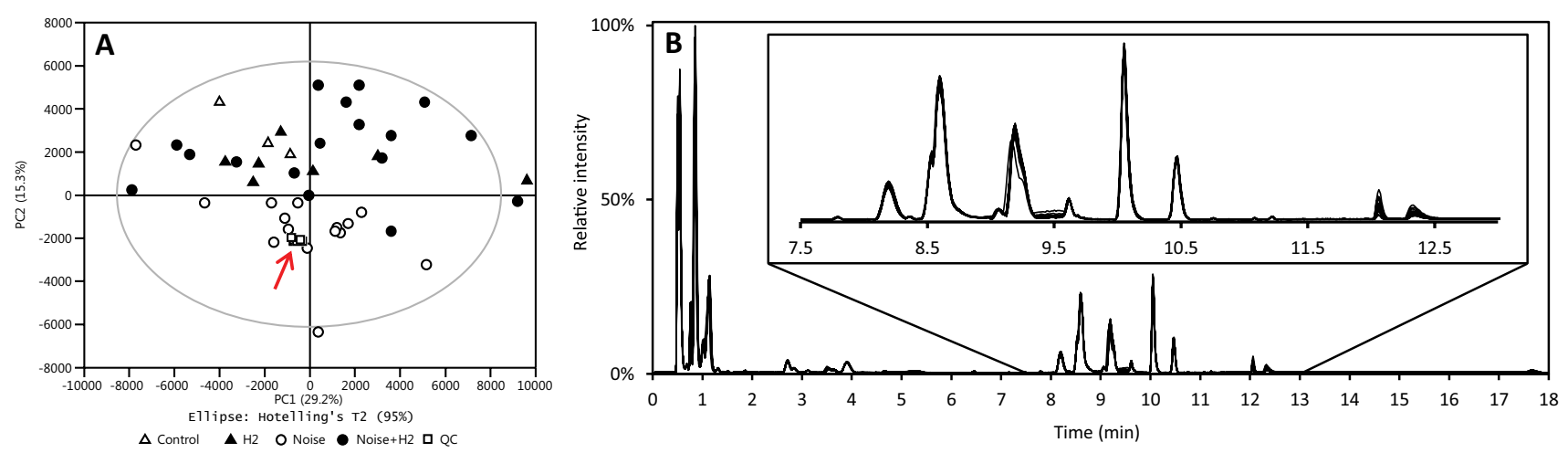

Fig. 1 Panel a: PCA scores plot of all study samples showing a clear clustering of the 15 repeated QC injections in the center of the first component indicated by the arrow. Panel $\mathbf{b}$ : commonly scaled and overlaid BPI traces of the 15 repeat QC injections

the only adduct being indicated by the model. In such cases, the feature was considered an artefact and was excluded.

For univariate analysis, the raw data was integrated using the TargetLynx module of MassLynx (Waters) and the peak integrals of each sample were normalized using the same normalization factor as in the data-preprocessing of the whole dataset.

\subsection{Univariate data analysis of selected features}

Significance testing of important features was performed using the Wilcoxon two-sided rank-sum test. Visualization of the relative peak areas of each sample group was done using box plots.

\section{Results and discussion}

\subsection{Quality control of LCMS analysis}

\subsubsection{Pre-analytical quality control}

The system suitability test described above showed that no clear systematic deviation was observed in relation to historical instrument response over time and it was concluded that the instrument was performing nominally.

In the conditioning step, a stable system should show convergence of retention times and peak areas in the targeted approach as well as convergence to a cluster in the first two components of the PCA scores plot. Prior to starting the analytical run, in positive mode the peak area RSD of the monitored compounds ranged from 0.83 to $9.57 \%$ and the
RSD of retention times ranged from 0.0 to $0.7 \%$ for all species. In negative mode the peak area RSD ranged from 1.88 to $12.16 \%$ and the RSD of retention times ranged from 0.00 to $0.39 \%$. The PCA scores plot of the conditioning injections appear to converge after ca 4-5 injections in positive mode, and after 2 injections in negative mode (see Figs. S1 and S2 in the Supplementary Material). Two of the seven compounds had RSD of peak areas $>10 \%$ in the negative conditioning (10.51\% and $12.16 \%$ for cytidine and tryptophan respectively) but showed a uniform residual distribution around the mean and it was concluded that the instrument was conditioned.

\subsubsection{Inter- and post-analytical quality control}

During the analytical runs, peak areas and retention times of the species used to assess conditioning were monitored successively in the QC sample injections. No sudden irregular shift in peak areas or retention times was observed in either positive or negative mode. Across all QC sample injections, RSD of peak areas and retention times was $3.4-12.3 \%$ and $0.04-0.34 \%$ respectively in positive mode and $3.4-13.3 \%$ and $0.02-0.08 \%$ respectively in negative mode. In Fig. 1a the first two components of the PCA scores plot of all study and QC sample injections is presented. The injections of the QC sample all cluster together tightly indicating that the technical variation in the dataset is low in contrast to the biological variation. The QC sample is a composite of all samples in the study which includes a number of sample groups not presented in this paper, this likely explains the slight offset of the cluster from the origin in the plot. 

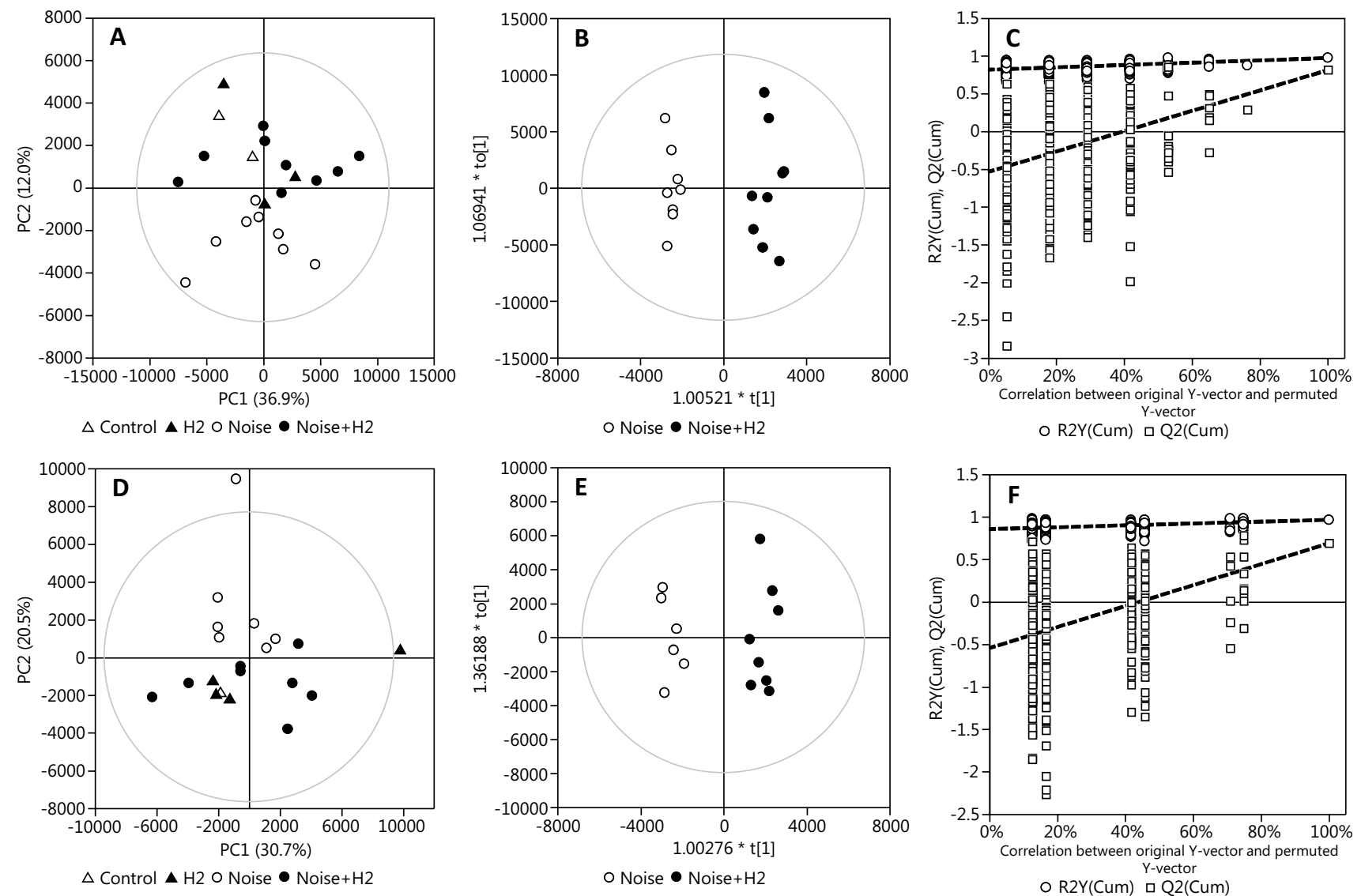

Fig. 2 Result of multivariate data analysis of samples from the noiseexposed, left ear and the contralateral, right ear. Panels a-c and d-f show the PCA scores plot, OPLS-DA scores plot, and permutation plot of the left and right ear, respectively. There is a clear separation of the Noise group from other groups in the metabolome of both ears. OPLS-DA models on Noise versus Noise $+\mathrm{H} 2$ exhibit excellent performance with a clear separation of the groups with no overlap (R2(cum) 0.968, Q2(cum) 0.809 and R2(cum) 0.963, Q2(cum) 0.686 in the left and right ear, respectively). Permutation tests (panels $\mathbf{c}$ and f shows left and right ear, respectively) showed intercepts $\mathrm{R} 2=0.815$, $\mathrm{Q} 2=-0.543$ in the left ear and $\mathrm{R} 2=0.855, \mathrm{Q} 2=-0.548$ in the right ear

\subsection{Multivariate data analysis and feature selection}

In total, 689 features matched into 276 pcgroups (ESI+) and 1023 features matched into 360 pcgroups (ESI-) were retained with a QC RSD $<30 \%$ and retention time between 60 and $800 \mathrm{~s}$.

Although the impulse noise were administered locally into the left ear canal, perilymph samples were taken from scala tympani of both ears and analyzed separately. It is clear from the PCA scores plots of the ESI(+)-data in Figs. 2a, d that there is a systematic difference in the perilymph metabolome of noise-exposed animals not treated (Noise, empty circles) and treated with $\mathrm{H}_{2}$ (Noise $+H 2$, filled circles) in 

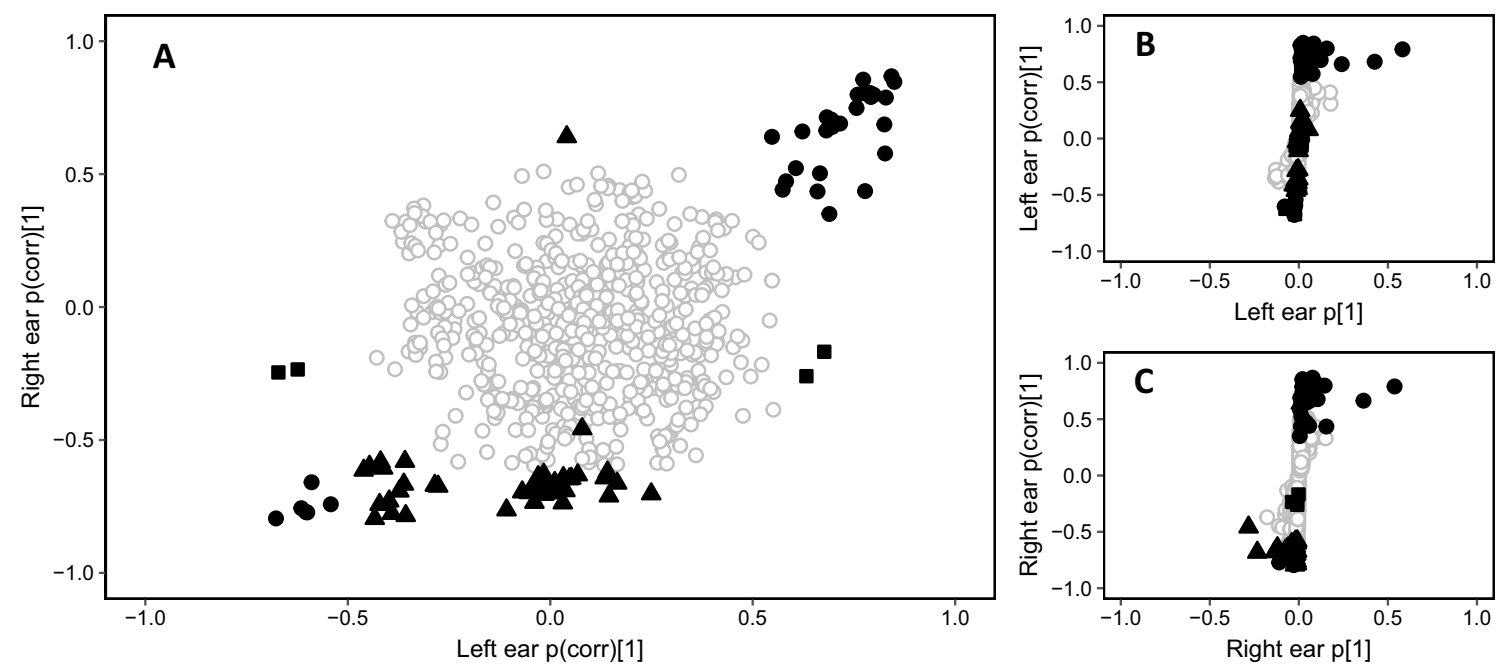

Both ears Unique left ear

Unique right ear $\bigcirc$ Non-selected feature

Fig. 3 Visualizations of feature predictive loadings as prepared by the SIMCA software. a SUS-plot of the two OPLS-DA models fitted using data from the left ear samples (x-axis) and right ear samples (y-axis). The plot shows the $\mathrm{p}$ (corr) value of the respective models as a scatter plot, facilitating the visualization of shared and unique struc- tures in the data. b, $\mathbf{c}$ shows the S-plot of the left and right sample models, respectively. These plots allow the selection of features with a high influence on the model (p[1], x-axis) and high correlation to group classification (p(corr)[1], y-axis). (Wiklund et al. 2008) the noise-exposed, left ear. A corresponding effect could also be observed in the contralateral, right ear. Considering the extreme intensity of the impulse noise, a significant effect can be expected also in the right ear, however boneconducted transmission of sound to the contralateral ear in guinea pigs is poorly investigated. Additionally, the result of the PCA indicate that the $\mathrm{H}_{2}$-treated noise-exposed animals are more similar in their metabolic profiles to the Control and $H 2$ groups than is the Noise group.

To investigate the effect of $\mathrm{H}_{2}$ on noise-exposed animals, OPLS-DA models of Noise versus Noise $+\mathrm{H} 2$ were fitted separately on samples from the left or right ears. In Fig. 2b, e are presented the results of models fitted using ESI(+)-data. Both models show a clear separation of the groups $(\mathrm{R} 2=0.968, \mathrm{Q} 2=0.809$ and $\mathrm{R} 2=0.963, \mathrm{Q} 2=0.686$ in the left and right ear respectively). The result of permutation tests $(n=500)$ is presented in Fig. 2c, $f$ with intercepts $\mathrm{R} 2 \mathrm{Y}=0.815$ and $\mathrm{Q} 2=-0.543$ in the left ear and $\mathrm{R} 2 \mathrm{Y}=0.855$ and $\mathrm{Q} 2=-0.548$ in the right ear showing that the models are reliable. CV-ANOVA tests of the two models yielded $p$-values $p<0.01$ and $p=0.057$ for the exposed-and non-exposed ear models respectively. The estimation of classification error by the LOOCV validation described above yielded a CCR of $94 \%$ and $87 \%$ for the left- and right ear models, respectively (see Supporting Information). No clear group discrimination was obtained using ESI(-)-data.

From the OPLS-DA models prepared using ESI(+)-data, a total of 85 features were selected (Fig. 3). Following manual verification as described above, 64 features were retained of which 27 were common to samples from both ears and 37 were unique to the right ear samples.

\subsection{Metabolite identification and univariate analysis}

The selected features were found to correspond to 15 individual chromatographic peaks (Table 1). Stachydrine, homostachydrine, pantothenic acid, creatine and a number of short-chain acyl carnitines could be putatively identified using the online databases HMDb (Wishart et al. 2007, 2009, 2013) and METLIN (Smith et al. 2005). Of these, the identities of stachydrine, creatine, acetylcarnitine, and butyrylcarnitine were confirmed using analytical standards based on retention time and MS/MS spectrum (see Table S3 and Figs. S3 to S19 in Supplementary Information). 
Table 1 The 15 metabolites selected as important to class discrimination by the multivariate models

\begin{tabular}{|c|c|c|c|c|c|}
\hline Metabolite & $\begin{array}{l}\text { MSI identifica- } \\
\text { tion level }^{\mathrm{a}}\end{array}$ & $\begin{array}{l}\text { Retention } \\
\text { time (s) }\end{array}$ & Prominent ions $(\mathrm{m} / \mathrm{z})^{\mathrm{b}}$ & $\begin{array}{l}\text { Main ion putative } \\
\text { molecular formula }^{\mathrm{c}}\end{array}$ & Pubchem CID \\
\hline U137 & 4 & 137 & $\begin{array}{l}156.845,158.843^{\mathrm{e}}, 394.606,396.604,398.602 \\
514.487,516.484,736.274\end{array}$ & $\mathrm{n} / \mathrm{d}^{\mathrm{d}}$ & \\
\hline U169 & 4 & 169 & 253.101 & {$\left[\mathrm{C}_{12} \mathrm{H}_{17} \mathrm{~N}_{2} \mathrm{O}_{2} \mathrm{~S}\right]+$} & \\
\hline Pantothenic acid & 2 & 173 & $90.056,220.118^{\mathrm{e}}, 296.0302$ & {$\left[\mathrm{C}_{9} \mathrm{H}_{18} \mathrm{NO}_{5}\right]+$} & 6613 \\
\hline Butyrylcarnitine & 1 & 450 & 232.154 & {$\left[\mathrm{C}_{11} \mathrm{H}_{22} \mathrm{NO}_{4}\right]+$} & 213144 \\
\hline Homostachydrine & 2 & 493 & $98.0966,158.118^{\mathrm{e}}, 160.123,180.1,196.073$ & {$\left[\mathrm{C}_{8} \mathrm{H}_{16} \mathrm{NO}_{2}\right]+$} & 441447 \\
\hline Stachydrine & 1 & 513 & $84.081,102.055,144.103^{\mathrm{e}}, 166.084,182.058$ & {$\left[\mathrm{C}_{7} \mathrm{H}_{14} \mathrm{NO}_{2}\right]+$} & 115244 \\
\hline U515 & 4 & 515 & 283.164 & {$\left[\mathrm{C}_{12} \mathrm{H}_{24} \mathrm{~N}_{2} \mathrm{O}_{4} \mathrm{Na}\right]+$} & \\
\hline U539 & 4 & 539 & $101.060,160.134^{\mathrm{e}}$ & {$\left[\mathrm{C}_{8} \mathrm{H}_{18} \mathrm{NO}_{2}\right]+$} & \\
\hline U547 & 4 & 547 & $116.047,138.055^{\mathrm{e}}, 160.037$ & {$\left[\mathrm{C}_{7} \mathrm{H}_{8} \mathrm{NO}_{2}\right]+$} & \\
\hline Acetylcarnitine & 1 & 554 & 204.124 & {$\left[\mathrm{C}_{9} \mathrm{H}_{18} \mathrm{NO}_{4}\right]+$} & 7045767 \\
\hline U569 & 4 & 569 & 262.165 & {$\left[\mathrm{C}_{12} \mathrm{H}_{24} \mathrm{NO}_{5}\right]+$} & \\
\hline U576 & 4 & 576 & $160.097,182.080^{\mathrm{e}}$ & {$\left[\mathrm{C}_{7} \mathrm{H}_{13} \mathrm{NO}_{3} \mathrm{Na}\right]+$} & \\
\hline U582*acylcarnitine & 3 & 582 & $211.058,248.150^{\mathrm{e}}$ & {$\left[\mathrm{C}_{11} \mathrm{H}_{22} \mathrm{NO}_{5}\right]+$} & \\
\hline Creatine & 1 & 628 & $90.0554,132.077^{\mathrm{e}}$ & {$\left[\mathrm{C}_{4} \mathrm{H}_{10} \mathrm{~N}_{3} \mathrm{O}_{2}\right]+$} & 586 \\
\hline U633 *acylcarnitine & 3 & 633 & 262.128 & {$\left[\mathrm{C}_{11} \mathrm{H}_{20} \mathrm{NO}_{6}\right]+$} & \\
\hline
\end{tabular}

Qualitative data shown is the identification level as specified by the MSI, retention time in seconds, prominent mass peaks in the spectrum of the peak, and a putative molecular formula

${ }^{a}$ Metabolite identification level as described by the metabolomics standardization initiative (MSI, Sumner et al. 2007)

${ }^{\mathrm{b}}$ Ions determined to be part of spectrum

${ }^{c}$ Determined using the iFit algorithm of the elemental composition tool in MassLynx (ver. 4.1, Waters) and selection of the formula with the highest iFit value

${ }^{\mathrm{d}}$ Could not be determined due to the complexity of the spectrum

${ }^{\mathrm{e}}$ Base peak

*Putatively determined compound class

The most intense mass peak of each metabolite was subjected to further univariate consideration and fold changes, VIPpred value, as well as the $\mathrm{p}$ value from the univariate significance tests are presented in Table 2. In Fig. 4, boxplots of peak intensities of the most intense mass peak from each metabolite are presented. In short, when contrasting the Noise and Noise $+\mathrm{H} 2$ groups, seven metabolites exhibit a different relative intensity in the samples from both ears whereas the other eight only show a corresponding effect in the samples from the right ears only. Stachydrine, homostachydrine, U169, U515, and U576 all exhibit a lower relative intensity in the Noise group in contrast to the Noise $+\mathrm{H} 2$ group in both ears, whereas a higher relative intensity is observed for U539, and U547 in both ears. Incidentally, the relative intensity of $\mathrm{U} 169$ in the $\mathrm{H} 2$ and Noise $+\mathrm{H} 2$ group is higher than in both the Control and Noise groups indicating that this is a potential effect of the $\mathrm{H}_{2}$ treatment. However, the small number of control group samples (Control and $\mathrm{H2}$ ) makes this conclusion tentative.

An additional eight metabolites were found to have a differentiating relative intensity in only the right ear samples. Pantothenic acid, creatine, butyrylcarnitine, acetylcarnitine, two unidentified acylcarnitines, U137, and U569 all had a higher relative intensity in the Noise group in contrast to the Noise $+H 2$ group.

\subsection{Biological interpretation}

\subsubsection{Noise-induced hearing loss}

Elevated levels of ROS follow the formation of excess amounts of superoxide inside the mitochondria of the hair cells via a few identified pathways (Henderson et al. 2006): (i) overdriving of the mitochondria due to noise stimulation, 
Table 2 Results of the univariate analysis of the main ions of each metabolite spectrum

\begin{tabular}{|c|c|c|c|c|c|c|c|c|}
\hline \multirow[t]{2}{*}{ Metabolite } & \multirow[t]{2}{*}{$\mathrm{QC} \mathrm{RSD}^{\mathrm{a}}(\%)$} & \multirow[t]{2}{*}{$\mathrm{m} / \mathrm{z}^{\mathrm{b}}$} & \multicolumn{2}{|c|}{ VIPpred $^{\mathrm{c}}$} & \multicolumn{2}{|c|}{ Fold change $^{\mathrm{d}}$} & \multicolumn{2}{|l|}{ p-value $e^{e}$} \\
\hline & & & Left ear & Right ear & Left ear & Right ear & Left ear & Right ear \\
\hline U137 & 6.1 & 157.845 & 0.33 & 0.81 & 1.15 & 1.35 & $4.8 \mathrm{E}-01$ & $3.7 \mathrm{E}-03$ \\
\hline U169 & 1.8 & 253.101 & 6.27 & 4.04 & 0.44 & 0.51 & $2.1 \mathrm{E}-02$ & $1.5 \mathrm{E}-01$ \\
\hline Pantothenic acid & 5.7 & 220.118 & 0.06 & 0.92 & 0.98 & 1.67 & $8.2 \mathrm{E}-01$ & $5.9 \mathrm{E}-03$ \\
\hline Butyrylcarnitine & 2.8 & 232.154 & 0.14 & 1.63 & 0.97 & 1.70 & $9.6 \mathrm{E}-01$ & $4.0 \mathrm{E}-02$ \\
\hline Homostachydrine & 3.1 & 158.118 & 11.10 & 9.48 & 0.36 & 0.46 & $7.9 \mathrm{E}-03$ & $9.3 \mathrm{E}-03$ \\
\hline Stachydrine & 2.8 & 144.103 & 15.16 & 14.04 & 0.27 & 0.33 & $2.5 \mathrm{E}-03$ & $3.1 \mathrm{E}-04$ \\
\hline U515 & 16.8 & 283.164 & 1.64 & 1.54 & 0.28 & 0.31 & $2.7 \mathrm{E}-02$ & $1.4 \mathrm{E}-02$ \\
\hline U539 & 2.8 & 160.134 & 2.07 & 2.88 & 2.83 & 4.29 & $3.6 \mathrm{E}-02$ & $3.1 \mathrm{E}-04$ \\
\hline U547 & 7.6 & 138.055 & 0.41 & 0.50 & 1.43 & 1.44 & $4.6 \mathrm{E}-02$ & $2.9 \mathrm{E}-02$ \\
\hline Acetylcarnitine & 3.0 & 204.124 & 1.42 & 7.38 & 0.94 & 1.82 & $1.0 \mathrm{E}+00$ & $3.7 \mathrm{E}-03$ \\
\hline U569 & 3.5 & 262.165 & 0.32 & 1.25 & 0.83 & 1.80 & $7.4 \mathrm{E}-01$ & $5.9 \mathrm{E}-03$ \\
\hline U576 & 9.1 & 182.080 & 0.66 & 0.51 & 0.55 & 0.63 & $1.5 \mathrm{E}-02$ & $1.5 \mathrm{E}-01$ \\
\hline U582 *acylcarnitine & 3.7 & 248.150 & 0.60 & 1.97 & 0.77 & 2.05 & $6.1 \mathrm{E}-01$ & $5.9 \mathrm{E}-03$ \\
\hline Creatine & 2.6 & 132.077 & 0.27 & 6.10 & 1.02 & 1.56 & $8.9 \mathrm{E}-01$ & $3.7 \mathrm{E}-03$ \\
\hline U633 *acylcarnitine & 6.5 & 262.128 & 0.02 & 0.71 & 1.05 & 1.65 & $8.9 \mathrm{E}-01$ & $1.2 \mathrm{E}-03$ \\
\hline
\end{tabular}

Presented is the RSD of peak intensities across the 15 QC sample injections, $\mathrm{m} / \mathrm{z}$ of the peak used for the analysis, VIPpred values determined from the OPLS-DA models, fold changes between groups Noise and Noise $+\mathrm{H} 2$, and p-value determined from a Wilcoxon rank-sum test of Noise versus Noise $+\mathrm{H} 2$

${ }^{a}$ Peak area RSD over QC sample injections

${ }^{\mathrm{b}} \mathrm{m} / \mathrm{z}$ of ion used for univariate analysis

${ }^{\mathrm{c}}$ Variable of importance in the predictive component of the OPLS-DA model

${ }^{\mathrm{d}}$ Fold change calculated as mean peak area in Noise sample group divided by mean peak area in Noise $+\mathrm{H} 2$ sample group

${ }^{\mathrm{e}}$ Two-sided wilcoxon rank-sum test of Noise versus Noise $+\mathrm{H} 2$

*Putatively determined compound class

(ii) excitotoxic activity by excessive neurotransmitter release into the hair cell synapses initiating apoptotic cell death further straining aerobic respiration, and (iii) a reduction in cochlear blood flow following noise exposure leading to hypoxia that lowers reaction efficiencies inside the mitochondria further exacerbating superoxide formation. Animals receiving $\mathrm{H}_{2}$ treatment (Noise $+\mathrm{H}_{2}$ group) after impulse noise exposure exhibited less hearing loss than animals in the Noise group (Videhult Pierre et al. Hydrogen gas inhalation attenuates acute noise trauma. A preclinical in vivo study, in manuscript). Thus, inhalation of $\mathrm{H}_{2}$ immediately after impulse noise trauma had a rescue effect on the cochlea and most probably on the cytotoxic mechanisms induced by ROS. As we have shown, this protective effect can also be seen in the perilymph metabolome. As the animals in the present study were allowed to survive only 4 days after impulse noise exposure the nature of hearing loss ought to be defined as acute auditory dysfunction.

\subsubsection{Changes in acyl carnitines}

The main role of acyl carnitines inside the cell is to facilitate transport of long-chain fatty acids across the mitochondrial membrane for subsequent beta-oxidation and energy production (Bieber and Choi 1977; Bender 2012; Longo et al. 2016; Traina 2016). In addition, acyl carnitines may also be formed in the catabolism of amino acids. As an example, the amino acids valine, isoleucine, and leucine all undergo catabolism that leads to acyl-CoA substrates that may form acyl carnitines (Bieber and Choi 1977; Violante et al. 2013). As has been shown, the levels of various acyl carnitines in the perilymph metabolome of the Noise group was higher than the levels observed in samples from the Noise $+\mathrm{H} 2$, Control, and $H 2$ groups. The increased levels of acyl carnitines are indicative of an increase in oxidative stress, which indicates that the metabolic strain caused by the noise exposure is attenuated by the administration of $\mathrm{H}_{2}$. 

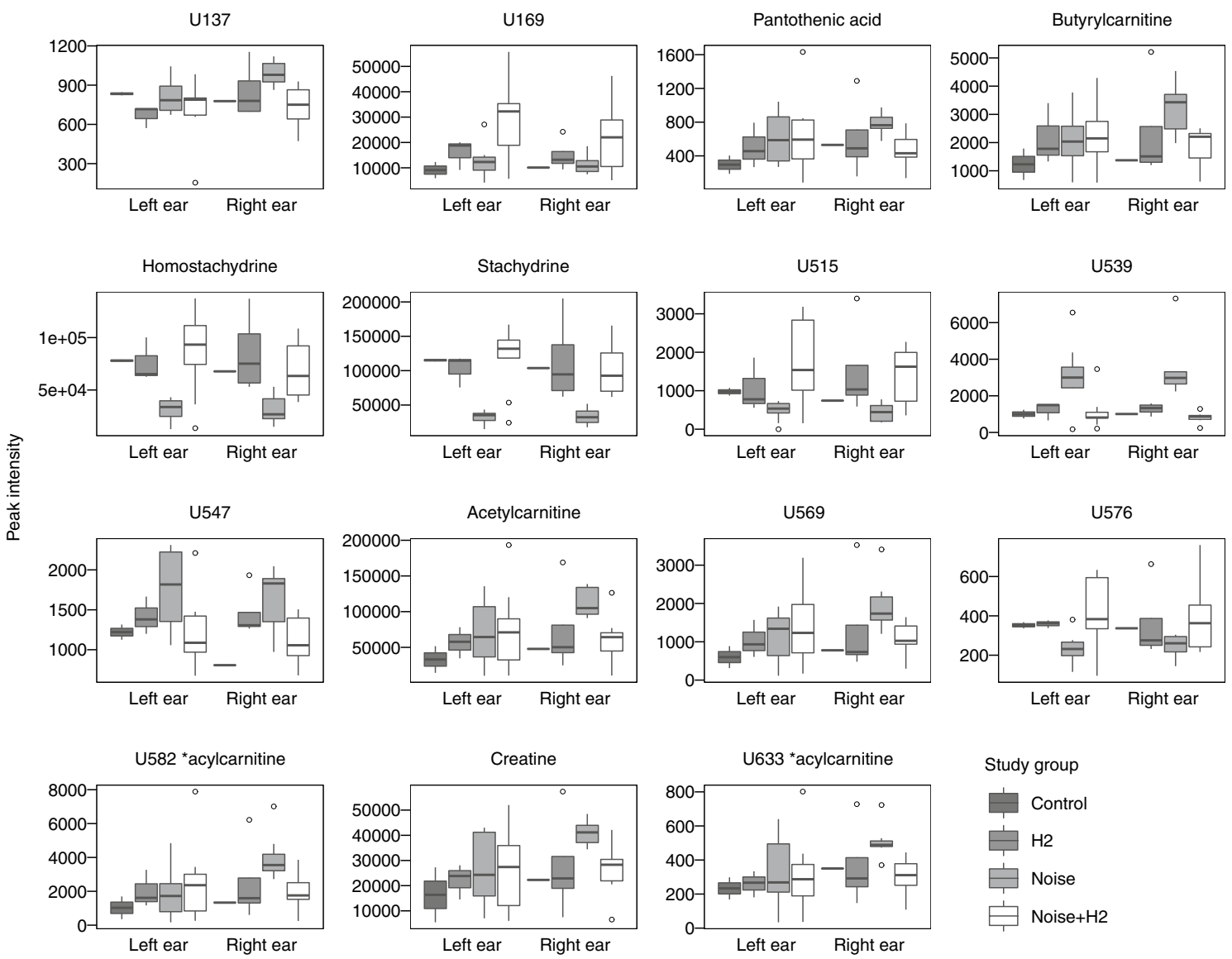

Fig. 4 Boxplots of main ion peak intensities of the selected metabolites in the Noise (exposed ear: $n=8$, unexposed ear: $n=7$ ), Noise $+\mathrm{H} 2$ (exposed ear: $n=9$, unexposed ear: $n=8$ ), Control

\subsubsection{Changes in stachydrine and homostachydrine}

Stachydrine and homostachydrine belong to a group of compounds known as osmoprotectants that help regulate cell volume (Lang 2007). Stachydrine is known to act as an osmoprotectant for E. coli and other bacteria in human urine and has also been detected in human blood (Chambers and Kunin 1987). However, to the best of our knowledge, no pathway for the formation of stachydrine or homostachydrine in mammals is known. They are a common component of many plants and their occurrence in mammals is likely explained by intake of such plants as food (Lever et al. 1994). The lower relative intensity of stachydrine and homostachydrine in the Noise group in contrast with the Noise $+\mathrm{H} 2$, Control, and $\mathrm{H} 2$ groups indicate the presence of osmolytic responses in the absence of $\mathrm{H}_{2}$ treatment. The (exposed ear: $\mathrm{n}=2$, unexposed ear: $\mathrm{n}=1$ ), and $\mathrm{H} 2$ (exposed ear: $\mathrm{n}=3$, unexposed ear: $n=4$ ) sample groups. Outlying samples are indicated by open circles

Noise $+\mathrm{H} 2$ group is again similar to the Control and $\mathrm{H} 2$ groups, which show that the $\mathrm{H}_{2}$ attenuate the effects of the noise exposure. Incidentally, stachydrine and other osmoprotectants have not been previously shown to have a roll in hearing loss.

\section{Conclusions}

An LCMS-based method has been presented for untargeted metabolomics of perilymph applied to samples taken from a guinea pig model of $\mathrm{H}_{2}$ attenuation of NIHL. Furthermore, it has been shown that the protocol is fit-for-purpose as the combination of targeted and untargeted quality control methods indicate a low analytical variation, as indicated 
by the low RSD in both peak areas and retention times as well as the tight clustering of the QC samples in the unsupervised data analysis. Supervised analysis of the obtained data afforded robust and highly discriminatory models indicating a number of metabolites as important including two osmoprotectants, a number of short-chain acyl carnitines and a number of other metabolites. Changes in the perilymph metabolome were found in samples taken from both ears indicating a bilateral effect, despite the local administration of noise into the left ear. For the most part, these effects appeared to originate from dysregulation in response to noise exposure as the $\mathrm{H}_{2}$-treated group appeared more similar in the models to the animals not exposed to noise. Thus, the results indicated that the increased strain on aerobic respiration caused by the noise exposure was attenuated by $\mathrm{H}_{2}$.

Acknowledgments Open access funding provided by Uppsala University.

Author contributions GL and PVP conceived and designed the research. KP, CP, TA, JH, and ME designed the metabolomics protocol and $\mathrm{KP}$ conducted the metabolomics analysis under supervision of $\mathrm{CP}$, TA, and MH. KP wrote the manuscript with contribution of knowledge and expertise from all authors. All authors read and approved the manuscript.

Funding This study was supported by Grants from Afa Insurance (dnr 110079), Foundation Tysta Skolan, Uppsala University Hospital ALFGrants, and funding from the Disciplinary domain of Medicine and Pharmacy, Uppsala University.

\section{Compliance with ethical standards}

Conflict of interest All authors declare that they have no conflict of interest.

Ethical approval All animal procedures were performed in accordance with the ethical guidelines of Uppsala University and consistent with national regulations for animal care and use (ethical permit C 106/13; Uppsala's ethical committee on animal experiments).

Data availability The metadata and scripts produced in the study are made available via https://github.com/krispir/NIHL_H2.

Open Access This article is distributed under the terms of the Creative Commons Attribution 4.0 International License (http://creativeco mmons.org/licenses/by/4.0/), which permits unrestricted use, distribution, and reproduction in any medium, provided you give appropriate credit to the original author(s) and the source, provide a link to the Creative Commons license, and indicate if changes were made.

\section{References}

Bender, D. A. (2012). Amino acid metabolism. Chichester: John Wiley \& Sons.

Bieber, L. L., \& Choi, Y. R. (1977). Isolation and identification of aliphatic short-chain acylcarnitines from beef heart: Possible role for carnitine in branched-chain amino acid metabolism. Proceedings of the National Academy of Sciences, 74(7), 2795-2798. https:// doi.org/10.1073/pnas.74.7.2795.

Bobbin, R. P., \& Fallon, M. (1992). Intense sound increases the level of an unidentified amine found in perilymph. Hearing Research, 63(1-2), 157-162. https://doi.org/10.1016/0378-5955(92)90082 $-\mathrm{X}$.

Chambers, S. T., \& Kunin, C. M. (1987). Isolation of glycine betaine and proline betaine from human urine. Assessment of their role as osmoprotective agents for bacteria and the kidney. Journal of Clinical Investigation, 79(3), 731-737. https://doi.org/10.1172/ jci112878.

Dieterle, F., et al. (2006). Probabilistic quotient normalization as robust method to account for dilution of complex biological mixtures. Application in $1 \mathrm{H}$ NMR metabonomics. Analytical Chemistry, 78(13), 4281-4290. https://doi.org/10.1021/ac051632c.

Drescher, M. J., Medina, J. E., \& Drescher, D. G. (1981). High-resolution analysis of physiological amino acids and related compounds in ten-microliter samples of guinea pig perilymph by the use of high-performance liquid chromatography. Analytical Biochemistry, 116(2), 280-286. https://doi.org/10.1016/00032697(81)90357-2.

Edvardsson Rasmussen, J., et al. (2018). The proteome of perilymph in patients with vestibular schwannoma. A possibility to identify biomarkers for tumor associated hearing loss? PLoS ONE, 13(6), e0198442. https://doi.org/10.1371/journal.pone.0198442.

Eriksson, L., Trygg, J., \& Wold, S. (2008). CV-ANOVA for significance testing of PLS and OPLS ${ }^{\circledR}$ models. Journal of Chemometrics, 22(11-12), 594-600. https://doi.org/10.1002/cem.1187.

Fransson, A. E., et al. (2017). Hydrogen inhalation protects against ototoxicity induced by intravenous cisplatin in the guinea pig. Frontiers in Cellular Neuroscience. https://doi.org/10.3389/fncel .2017 .00280 .

Fujita, T., et al. (2015). Metabolomic profiling in inner ear fluid by gas chromatography/mass spectrometry in guinea pig cochlea. $\mathrm{Neu}$ roscience Letters, 606, 188-193. https://doi.org/10.1016/j.neule t.2015.09.001.

Gika, H. G., et al. (2007). Within-day reproducibility of an HPLC-MSbased method for metabonomic analysis: Application to human urine. Journal of Proteome Research, 6(8), 3291-3303. https:// doi.org/10.1021/pr070183p.

Hara, A., Salt, A. N., \& Thalmann, R. (1989). Perilymph composition in scala tympani of the cochlea: Influence of cerebrospinal fluid. Hearing Research, 42(2-3), 265-271. https://doi. org/10.1016/0378-5955(89)90150-0.

Hellberg, V., et al. (2013). Cochlear pharmacokinetics of cisplatin: An in vivo study in the guinea pig. The Laryngoscope, 123(12), 3172-3177. https://doi.org/10.1002/lary.24235.

Henderson, D., et al. (2006). The role of oxidative stress in noiseinduced hearing loss. Ear and Hearing, 27(1), 1-19. https://doi. org/10.1097/01.aud.0000191942.36672.f3.

Huang, Y., et al. (2011). Beneficial effects of hydrogen gas against spinal cord ischemia-reperfusion injury in rabbits. Brain Research, 1378, 125-136. https://doi.org/10.1016/j.brainres.2010.12.071.

Kei-Fukuda, K., et al. (2007). Inhalation of hydrogen gas suppresses hepatic injury caused by ischemia/reperfusion through reducing oxidative stress. Biochemical and Biophysical Research Communications, 361(3), 670-674. https://doi.org/10.1016/j. bbrc.2007.07.088.

Kikkawa, Y. S., et al. (2014). Hydrogen protects auditory hair cells from cisplatin-induced free radicals. Neuroscience Letters, 579, 125-129. https://doi.org/10.1016/j.neulet.2014.07.025.

Kuhl, C., Tautenhahn, R. and Neumann, S. (2014) LC-MS Peak Annotation and Identification with CAMERA, October.

Kuhl, C., et al. (2012). CAMERA: An integrated strategy for compound spectra extraction and annotation of liquid chromatography/mass 
spectrometry data sets. Analytical Chemistry, 84(1), 283-289. https://doi.org/10.1021/ac202450g.

Kurioka, T., et al. (2014). Inhaled hydrogen gas therapy for prevention of noise-induced hearing loss through reducing reactive oxygen species. Neuroscience Research, 89, 69-74. https://doi. org/10.1016/j.neures.2014.08.009.

Lang, F. (2007). Mechanisms and significance of cell volume regulation. Journal of the American College of Nutrition, 26(sup5), 613S-623S. https://doi.org/10.1080/07315724.2007.10719667.

Le, T. N., et al. (2017). Current insights in noise-induced hearing loss: A literature review of the underlying mechanism, pathophysiology, asymmetry, and management options. Journal of Otolaryngology - Head \& Neck Surgery, 46(1), 41. https://doi.org/10.1186/ s40463-017-0219-x.

Leary Swan, E. E., et al. (2009). Proteomics analysis of perilymph and cerebrospinal fluid in mouse. The Laryngoscope, 119(5), 953-958. https://doi.org/10.1002/lary.20209.

Lever, M., et al. (1994). Glycine betaine and proline betaine in human blood and urine. Biochimica et Biophysica Acta (BBA) - General Subjects, 1200(3), 259-264. https://doi.org/10.1016/03044165(94)90165-1.

Libiseller, G., et al. (2015). IPO: A tool for automated optimization of XCMS parameters. BMC Bioinformatics, 16(1), 118. https://doi. org/10.1186/s12859-015-0562-8.

Lin, Y., et al. (2011). Hydrogen in drinking water attenuates noiseinduced hearing loss in guinea pigs. Neuroscience Letters, 487(1), 12-16. https://doi.org/10.1016/j.neulet.2010.09.064.

Longo, N., Frigeni, M., \& Pasquali, M. (2016). Carnitine transport and fatty acid oxidation. Biochimica et Biophysica Acta (BBA) - Molecular Cell Research, 1863(10), 2422-2435. https://doi. org/10.1016/j.bbamcr.2016.01.023.

Lysaght, A. C., et al. (2011). Proteome of human perilymph. Journal of Proteome Research, 10(9), 3845-3851. https://doi.org/10.1021/ pr200346q.

Mavel, S., et al. (2018). Validation of metabolomics analysis of human perilymph fluid using liquid chromatography-mass spectroscopy. Hearing Research, 367, 129-136. https://doi.org/10.1016/j.heare s.2018.05.016.

Medina, J. E., \& Drescher, D. G. (1981). The amino-acid content of perilymph and cerebrospinal fluid from guinea-pigs and the effect of noise on the amino-acid composition of perilymph. Neuroscience, 6(3), 505-509. https://doi.org/10.1016/0306-4522(81)90142 -1 .

Palmer, J. C., et al. (2018). Comparing perilymph proteomes across species. The Laryngoscope, 128(1), E47-E52. https://doi. org/10.1002/lary.26885.

Psychogios, N., et al. (2011). The human serum metabolome. PLoS ONE, 6(2), e16957. https://doi.org/10.1371/journal.pone.00169 57.

$\mathrm{Qu}, \mathrm{J}$., et al. (2012). Inhalation of hydrogen gas attenuates cisplatininduced ototoxicity via reducing oxidative stress. International Journal of Pediatric Otorhinolaryngology, 76(1), 111-115. https ://doi.org/10.1016/j.ijporl.2011.10.014.

Sangster, T., et al. (2006). A pragmatic and readily implemented quality control strategy for HPLC-MS and GC-MS-based metabonomic analysis. The Analyst, 131(10), 1075-1078. https://doi. org/10.1039/b604498k.

Scheibe, F., \& Haupt, H. (1985). Biochemical differences between perilymph, cerebrospinal fluid and blood plasma in the guinea pig.
Hearing Research, 17(1), 61-66. https://doi.org/10.1016/03785955(85)90131-5.

Schmitt, H. A., et al. (2017). Proteome analysis of human perilymph using an intraoperative sampling method. Journal of Proteome Research, 16(5), 1911-1923. https://doi.org/10.1021/acs.jprot eome.6b00986.

Sha, S.-H., \& Schacht, J. (2017). Emerging therapeutic interventions against noise-induced hearing loss. Expert Opinion on Investigational Drugs, 26(1), 85-96. https://doi.org/10.1080/13543 784.2017.1269171.

Smith, C. A., et al. (2005). METLIN: A metabolite mass spectral database. Therapeutic Drug Monitoring, 27(6), 747-751.

Smith, C. A., et al. (2006). XCMS: Processing mass spectrometry data for metabolite profiling using nonlinear peak alignment, matching, and identification. Analytical Chemistry, 78(3), 779-787. https:// doi.org/10.1021/ac051437y.

Stanstrup, J., et al. (2013). Metabolite profiling and beyond: Approaches for the rapid processing and annotation of human blood serum mass spectrometry data metabolomics and metabolite profiling. Analytical and Bioanalytical Chemistry, 405(15), 5037-5048. https://doi.org/10.1007/s00216-013-6954-6.

Sumner, L. W., et al. (2007). Proposed minimum reporting standards for chemical analysis. Metabolomics, 3(3), 211-221. https://doi. org/10.1007/s11306-007-0082-2.

Traina, G. (2016). The neurobiology of acetyl-L-carnitine. Frontiers in Bioscience, 21(7), 4459. https://doi.org/10.2741/4459.

Veselkov, K. A., et al. (2011). Optimized preprocessing of ultraperformance liquid chromatography/mass spectrometry urinary metabolic profiles for improved information recovery. Analytical Chemistry, 83(15), 5864-5872. https://doi.org/10.1021/ac201 065j.

Violante, S., et al. (2013). Substrate specificity of human carnitine acetyltransferase: Implications for fatty acid and branched-chain amino acid metabolism. Biochimica et Biophysica Acta (BBA) - Molecular Basis of Disease, 1832(6), 773-779. https://doi. org/10.1016/j.bbadis.2013.02.012.

Wiklund, S., et al. (2008). Visualization of GC/TOF-MS-based metabolomics data for identification of biochemically interesting compounds using OPLS class models. Analytical Chemistry, 80(1), 115-122. https://doi.org/10.1021/ac0713510.

Wishart, D. S., et al. (2007). HMDB: The human metabolome database. Nucleic Acids Research, 35(SUPPL. 1), 521-526. https:// doi.org/10.1093/nar/gk1923.

Wishart, D. S., et al. (2009). HMDB: A knowledgebase for the human metabolome. Nucleic Acids Research, 37(SUPPL. 1), 603-610. https://doi.org/10.1093/nar/gkn810.

Wishart, D. S., et al. (2013). HMDB 3.0-The human metabolome database in 2013. Nucleic Acids Research, 41(D1), 801-807. https ://doi.org/10.1093/nar/gks1065.

$\mathrm{Wu}, \mathrm{Y} .$, \& Li, L. (2016). Sample normalization methods in quantitative metabolomics. Journal of Chromatography A, 1430, 80-95. https ://doi.org/10.1016/j.chroma.2015.12.007.

Publisher's Note Springer Nature remains neutral with regard to jurisdictional claims in published maps and institutional affiliations. 